
\title{
25 Research Square \\ Differentially expressed liver exosome-related genes as a candidate prognostic biomarker for hepatocellular carcinoma
}

\section{Bangyou Zuo}

Peking Union Medical College Hospital

Haitao Zhao ( $\sim$ ZhaoHT@pumch.cn)

PUMCH: Peking Union Medical College Hospital https://orcid.org/0000-0002-3444-8044

Jin Bian

PUMCH: Peking Union Medical College Hospital

Junyun Long

PUMCH: Peking Union Medical College Hospital

\section{Xu Yang}

PUMCH: Peking Union Medical College Hospital

Bo Hu

PUMCH: Peking Union Medical College Hospital

\section{Xiaobo Yang}

PUMCH: Peking Union Medical College Hospital

\section{Xinting Sang}

PUMCH: Peking Union Medical College Hospital

\section{Primary research}

Keywords: Hepatocellular carcinoma (HCC), Exosome, Prognostic model, Immune microenvironment, Immunotherapy

Posted Date: November 17th, 2020

DOl: https://doi.org/10.21203/rs.3.rs-105334/v1

License: (c) (i) This work is licensed under a Creative Commons Attribution 4.0 International License. Read Full License 


\section{Abstract \\ Background}

The function of exosome includes cell-to-cell communication, neovascularization, and metastasis of cancer cell and drug resistance, which plays an important part in the occurrence and progression of hepatocellular carcinoma (HCC). Because the mechanism in this area is less studied, our goal is to identify exosome-related genes in HCC, establish a reliable prognostic model for liver cancer patients, and explore its underlying mechanisms.

\section{Methods}

The exoRbase database and The Cancer Genome Atlas (TCGA) database were used to analyze differentially expressed genes (DEGs). Cox regression and LASSO analysis were applied to determine DEGs closely related to overall survival (OS). Then the exosome-related prognostic model was constructed in TCGA and validated in the database of International Cancer Genome Consortium (ICGC). Nomogram graph was performed to predict the survival. CIBERSORT was used to estimate the score of different type of immune cells. DEGs related to immunotherapy are used to predict the effect of immunotherapy.

\section{Results}

48 exosome-related DEGs were obtained and five genes (XP01, IFI30, FBX016, CALM1, MORC3) among them were selected to construct predictive model. Then we divided the HCC patients into low-risk and high-risk groups by the best cut-off value according to the X-tile software. The high-risk related to exosome were significantly associated with a poor prognosis. Moreover, the features related to exosome could positively regulate immune response. At the same time, the proportion of $T$ cell regulatory factors (Tregs) and macrophages M2 is higher in the high-risk group, and high-risk group exhibited higher expression of immune checkpoint molecular including PD-L1, PD-L2, TIGIT, and ID01.

\section{Conclusions}

Overall, our research showed that markers related to exosomes were potential biomarkers for the prognosis of HCC, providing an immunological perspective for the development of precision treatment.

\section{Introduction}

Hepatocellular carcinoma (HCC) ranks the fourth leading cause of cancer death all over the world, accounting for 85 percent to 90 percent of primary liver cancer. It is estimated that there are approximately 841,000 new cases of HCC each year ${ }^{[1-2]}$. Despite new breakthroughs in target therapy, immunity 
therapy, interventional therapy, operational techniques and liver transplantation, the prognosis of HCC is

still poor due to the high metastasis rate and high recurrence rate. ${ }^{[3-6]}$ Traditional models use clinicopathological parameters such as stage and other parameters to forecast the prognosis of HCC. However, due to the heterogeneity, it is meaningful to study the underlying molecular and identify novel markers for diagnosis and prognosis.

Exosomes are tiny extracellular vesicles with a lipid bilayer membrane structure. They were first discovered by Johnstone in 1989 when he studied reticulocytes. Exosomes can transport biologically active molecules between cells through various biological molecules and various biological molecules in the cell, which can regulate the microenvironment between cells and the immune system. ${ }^{[3,7-8]}{ }^{2}$ Exosomes derived from tumor tissue contain numerous cancer-related biomarkers, such as miRNA, which can be used to detect early-stage HCC. Although some breakthroughs have been made in the field of exosome, the specific biological functions of exosome have not yet been fully elucidated. ${ }^{[9-12]}$ Therefore, we are supposed to comprehensively study the exosome-related genes in HCC which may help to predict the outcome of HCC and find potential new drug candidates for specific targeted therapies.

\section{Methods}

\subsection{Data collection}

The exosome-related genes of liver were downloaded from the exoRbase (http:// exorbase.org/database/). The expression of mRNA and corresponding clinical data were obtained from TCGA website. For the validation dataset, the data of genes expression were obtained from the LIRI-JP of ICGC database.

\subsection{Identification of DEGs between HCC and non-cancer tissues and $\mathrm{GO}$ enrichment}

DEGs related to exosome and liver cancer are identified with limma (a type of $\mathrm{R}$ package). The DEGs with adjusted $\mathrm{P}<0.05$ and $\log 2 \mathrm{FC}>1$ were further analyzed. In order to study the potential biological effects of exosome-related genes, GO enrichment analysis was carried out, namely cell composition (CC), molecular function (MF) and biological process (BP) reflecting the functional annotation of key genes.

\subsection{Competing endogenous network construction}

Follow the steps below to construct the HCC ceRNA network (1) Use the miRcode database (http://mircode.org/) to scientifically search for the interaction between IncRNA and miRNA and perform matching miRNA interactions; (2) The database of TargetScan (http://targetscan.org/) was used to predict miRNA targeted mRNA. (3) ENCORI database (http://starbase.sysu.edu.cn/) predicted the relationship between CircRNA-miRNA. (4) Only the mRNA that exists in these three databases and overlaps with the mRNA can construct a ceRNA network. Use Cytoscape 3.7.2 to obtain the result graph.

\subsection{Development and validation of a prognostic model}


First of all, we performed a univariate Cox regression analysis and determine that the $p$ value is less than 0.05 is meaningful to the DEGs associated with OS. Subsequently, the glmnet, a package of R software, was used to narrow the genetic screening range, and then the LASSO regression was carried out. ${ }^{[13]}$ We fulfilled 1,000 repetitions on the dataset and chose markers with a repetition greater than 500 . Next, we performed a multivariate Cox regression analysis to evaluate the strongly correlated genes, and then developed a prognostic model. From the multivariate Cox regression, we can get the regression coefficient $(\beta)$. The formula is as follows PI (prognostic index) $=(\beta$ mRNA1* the expression value of mRNA1) + $(\beta m R N A 2 *$ the expression value of mRNA2) $+\ldots+(\beta m R N A n *$ the expression value of mRNAn). The software of X-tile was employed to affirm the optimal cut-off value, and we divided patients into high-risk and low-risk groups according to the best cut-off value. The predictive power of the prognostic model was checked by Kaplan-Meier survival analysis, and the model was validated in ICGC database later.

\subsection{Identification of Independent Prognostic Parameters for HCC}

The independent factors affecting clinical prognosis were obtained by Univariate and multivariate Cox proportional hazards regression analysis. A two-sided $P$ value $<0.05$ was recognized as statistically meaningful, and then calculated the hazard ratio (HR) and confidence interval (Cl) of ninety-five percent.

\subsection{Establishment and evaluation of forecast nomogram}

Independent prognostic factors obtained from Cox analysis were used to construct a nomogram so that we can predict the 1-, 2-, and 3-year survival rates of HCC patients. We used calibration chart for internal verification of the nomogram, and time-varying ROC curve to assess the predictive ability. ${ }^{[14-15]}$

\subsection{Estimation of the immune cell types score}

CIBERSORT is a new way to characterize cell composition by genes expression profiling, which is a frequently used method for evaluating and analyzing the infiltration of immune cell. ${ }^{[16]}$ The difference of the ratio of immune-cell types between low-risk and high-risk populations was forecasted by CIBERSORT. The Genefilter package of R software was used to screen each sample, and determine that the $p$ value is less than 0.05 as meaningful.

\subsection{Evaluation of immunotherapy effect}

Immunotherapy is a very promising treatment method, and the therapeutic effect is related to molecules such as PD-L1, PD-L2, ID01 and TIGIT. Analyze the difference of expression of these molecules between the groups of low-risk and high-risk.

\section{Results}

\subsection{Competing endogenous network construction and GO enrichment analysis}


From the genes downloaded from the exoRbase database, we find the difference genes of mRNA, IncRNA, circRNA between the tumor and non-tumor tissues respectively. And then explored the relationships among mRNA, IncRNA, microRNA, circRNA, pairs of IncRNA and miRNA, miRNA and mRNA and circRNA and miRNA were used to develop ceRNA network by cytoscape. Totally, 398 pairs of IncRNAs-miRNAs, 681 pairs of miRNAs-mRNAs and 200 pairs of circRNAs-miRNAs were confirmed. Finally, 10 DElncRNAs, 72 DEmiRNAs, 48 DEmRNAs and 15DEcircRNAs were involved to construct ceRNA network.(Fig. 1A) To better understand the biological consequences of the exosome-related genes, we carried out GO enrichment analysis. The Go enrichment result illustrated that the exosome- related genes were significantly enriched in BP and MF, which were related to protein localization to nucleus and phosphatase regulator activity (Fig. 1B). All these signaling pathways are reported to take significant roles in the development of cancer. The results may indicate that abnormal expression of exosomerelated genes might influence the pathway activation and mitosis and further lead to HCC.

\subsection{Construction an exosome-related genes prognostic model in TCGA}

Intersect the mRNAs found in ceRNA network with TCGA genes, we got 48 DEGs. Then, we carried out a univariate Cox regression and 18 DEGs were found to be significantly correlated with OS in HCC patients $(P<0.01)$. And a lasso analysis was carried out to narrow down the gene range. We have selected 6 DEGs, which appeared more than 500 times of 1000 cycles totally. At last, a multivariate Cox regression was carried out, and five genes (XPO1, IFI30, FBX016, CALM1, MORC3) were chosen to establish the prognostic model as shown below: $\mathrm{PI}=(0.469$ * expression value of $\mathrm{XPO1})+(0.141$ * expression value of $\mathrm{IFI} 30)+\left(0.218^{*}\right.$ expression value of FBX016) + $\left(0.146^{*}\right.$ expression value of CALM1 $)+(-0.343$ * expression value of MORC3). The best cut-off value of 2.06 for the risk score was got by the software of $X$-tile. Then HCC patients were divided into low-risk and high-risk groups according to the best cut-off value. The overall survival of the high-risk group was significantly poorer than that of low-risk group according to the Kaplan-Meier curve $(P<0.001, H R=3.51)(F i g .2 A)$. The area under the time-dependent ROC curves (AUCs) for 1-, 2- and 3-year overall survival (OS) were $0.74,0.71,0.7$ and 0.68 respectively, indicating a good predictive performance of this prognostic model (Fig. $2 \mathrm{C}$ ).

\subsection{External validation of exosome-related genes prognostic model in ICGC}

Samples from ICGC were further used to validate the predictive power of this prognostic mode. Same way as above, patients were divided into low- and high-risk groups with the same cut-off value of 2.06 and the survival rate of HCC patients in low-risk group was higher than that in the high-risk group $(P=0.009, H R=$ 2.65) (Fig. 2B). And the AUCs of the five-gene prognostic model were $0.63,0.58$ and 0.63 for the 1-, 2- and 3-year survival times (Fig. 2D), which showed that this model is reliable.

\subsection{Establishment a predictive nomogram}


Univariate Cox regression analysis illustrated that T.N.M. stage and risk-score were both meaningful factors (Fig. 3A). Multivariate Cox regression analysis showed T.N.M. stage and risk-score were both independent prognostic factors (Fig. 3B).So, the nomogram was built by including T.N.M. stage and the risk score (Fig. 3C). Assign a score to the independent factors of each level, and calculate the total score by summing the scores of each person. By transforming the relationship of the total score, the survival rate of 1-, 2-and 3-years can be obtained. The calibration map was used for internal verification of this nomogram, which showed that there was a better conformity between the predicted results and the realistic observation results (Fig. 3D-F).

\subsection{Computation of immune cell type fractions}

A new tool called CIBERSORT is used to directly analyze the types and expression of immune cells in the tissue, combined with the Leukocyte signature matrix (LM22). And we assessed the differences of twenty-two types of immune cells between the two groups of HCC patients. And 2 immune cells ( $T$ cells regulatory, $\mathrm{P}=0.043$, Macrophages $\mathrm{M} 2, \mathrm{P}=0.048$ ) were different between the two groups (Fig. $4 \mathrm{~A})$. From our result, we may conclude that the poor prognosis relates to the high content of Tregs and M2. As shown in Fig. 4B, the twenty-two immune cell proportions between high-risk and low-risk groups were different, and the relationship among immune cells showed in Fig. 4C.

\subsection{Evaluation of the immunotherapy effect}

Blockade of immune checkpoints has been found to be a promising treatment method of many cancers. Subsequently, we studied the different expression of key immune checkpoint molecular including PD-L1, PD-L2, TIGIT and ID01. As shown in Figs. 5A-5D, high-risk HCC patients seemed to express higher immune checkpoint molecules than low-risk patients $(p<0.05)$. From our research, we may conclude that the poor prognosis is related to the high expression of exosomes-related genes.

\section{Discussion}

Hepatocellular carcinoma is a deadly malignant tumor and has been a considerable public health problem in many countries for many years. Although the evidence-based treatment of HCC has made great progress, its survival rate has not improved satisfaction. ${ }^{[17]}$ More and more evidences show that the malignant phenotype is affected by the cancer-related microenvironment. Hence, it is of great importance to discover immune bio-markers to forecast the prognosis of HCC patients, which may be helpful in immunotherapy. ${ }^{[18-20]}$

In our study, we built an exosome-related gene prognostic model to forecast the prognosis of HCC patient based on TCGA database, and validated the prediction ability in external database successfully. We confirmed that the prognostic model can divide HCC patients into low-risk and high-risk subtypes, and showed that the oncology outcome of low-risk subtypes is better than that of high-risk groups. Then a nomogram was built to predict the survival rate of 1-, 2- and 3-years. And from the calibration plot, we can 
find the red curve is very close to the real curve which means a better predictive value. So, we further analyzed the difference of infiltrating immune cells and immune-related genes expression between lowand high-risk groups. In our study, high-risk patients seem to be more likely to show higher immune checkpoint molecules and have a poor prognosis.

So far, a lot of work has focused on the function of exosome in intercellular communication of HCC. It has been widely accepted that HCC cells communicate with normal cells and promote the development and metastasis of HCC through exosome. Some of them regulate the signal transduction pathway among cells, while others can be used as medicine due to the protective effect of the outer membrane. [21-22] However, there are still few studies on the role of exosome in immunotherapy. Our article has done some exploratory research in this area and obtained some results, hoping to help future research.

Although our research had done a lot of work, there are still some limitations. First of all, our retrospective studies need to be further verified in prospective studies, especially RCTs. Second, after the process of lasso analysis some important factors that contribute to the prognosis of HCC may be ignored. In addition, the expression and prognostic effects of these five genes at the protein level need further research. And need to perform functional experiments to clarify its potential mechanism. Finally, the specific mechanism between tumor cells and immune cells needs to be further studied to clarify.

\section{Declarations}

\section{Ethics approval and consent to participate}

Not applicable.

\section{Consent for publication}

Not applicable.

\section{Availability of data and materials}

The data used to support the findings of this study are available from the TCGA and ICGC database.

\section{Competing interests}

The authors declare that they have no competing interests.

\section{Funding}

This work was supported by International Science and Technology Cooperation Projects (2016YFE0107100 and 2015DFA30650), CAMS Innovation Fund for Medical Science (CIFMS) (2017-I2M4-003 and 2018-I2M-3-001), Beijing Natural Science Foundation (L172055 and 7192158), the Fundamental Research Funds for the Central Universities(3332018032), National Ten-thousand Talent Program and Youth Innovation Promotion Association of the Chinese Academy of Sciences 
(N0.2016252), the Youth foundation of the national natural science foundation of China(Grant No.81902620), the Youth foundation of the national natural science foundation of China(Grant No.81902353).

\section{Acknowledgments}

Not applicable.

\section{Authors' contributions}

Conception and design: BZ and JL. Acquisition of data: BZ and JB. Analysis and interpretation of data: BZ and JL. Drafting the article: BZ, JB and JL. Critically revising the article: all authors. Study supervision: $\mathrm{HZ}$. All authors read and approved the final manuscript.

\section{References}

1. Global Burden of Disease Liver Cancer, Akinyemiju T, Abera S., et al., The Burden of Primary Liver Cancer and Underlying Etiologies From 1990 to 2015 at the Global, Regional, and National Level: Results From the Global Burden of Disease Study 2015. JAMA Oncol, 2017. 3(12): p. 16831691.

2. Villanueva A. Hepatocellular Carcinoma. N Engl J Med. 2019 Apr 11;380(15):1450-1462.

3. Chen R, Xu X, Tao Y, et al. Exosomes in hepatocellular carcinoma: a new horizon. Cell Commun Signal. 2019;17(1):1.

4. Llovet JM, Ricci S, Mazzaferro V, et al. Sorafenib in advanced hepatocellular carcinoma. N Engl J Med. 2008;359(4):378-90.

5. Cheng AL, Kang YK, Chen Z, et al. Efficacy and safety of sorafenib in patients in the Asia-Pacific region with advanced hepatocellular carcinoma: a phase III randomised, double-blind, placebocontrolled trial. Lancet Oncol. 2009 Jan;10(1):25-34.

6. Kudo M, Finn RS, Qin S, et al. Lenvatinib versus sorafenib in first-line treatment of patients with unresectable hepatocellular carcinoma: a randomised phase 3 non-inferiority trial. Lancet. 2018 Mar 24;391(10126):1163-1173.

7. Li L, Wang H. Heterogeneity of liver cancer and personalized therapy. Cancer Lett. 2016 Sep 1;379(2):191-7.

8. Roma-Rodrigues C, Raposo LR, Cabral R, et al. Tumor Microenvironment Modulation via Gold Nanoparticles Targeting Malicious Exosomes: Implications for Cancer Diagnostics and Therapy. Int J Mol Sci. 2017;18(1).

9. Chen G, Huang AC, Zhang W, et al. Exosomal PD-L1 contributes to immunosuppression and is associated with anti-PD-1 response. Nature. 2018;560(7718):382-6.

10. Melo SA, Sugimoto H, O'Connell JT, et al. Cancer exosomes perform cell-independent microRNA biogenesis and promote tumorigenesis. Cancer Cell. 2014;26(5):707-721. 
11. Li X, Li C, Zhang L, et al. The significance of exosomes in the development and treatment of hepatocellular carcinoma. Mol Cancer. 2020;19(1):1. Published 2020 Jan 4.

12. Miura K. The day will come to treat HCC in a drugstore? HepatoBiliary Surg Nutr. 2017;6(6):420-423.

13. Tibshirani R. The lasso method for variable selection in the Cox model. Stat Med. 1997 Feb 28;16(4):385-95.

14. Heagerty PJ, Lumley T, Pepe MS. Time-dependent ROC curves for censored survival data and a diagnostic marker. Biometrics. 2000 Jun;56(2):337-44.

15. Iasonos A, Schrag D, Raj GV, Panageas KS. How to build and interpret a nomogram for cancer prognosis. J Clin Oncol. 2008 Mar 10;26(8):1364-70.

16. Newman AM, Liu CL, Green MR, et al. Robust enumeration of cell subsets from tissue expression profiles. Nat Methods. 2015 May;12(5):453-7.

17. Bruix, J., M. Reig, and M. Sherman, Evidence-Based Diagnosis, Staging, and Treatment of 330 Patients With Hepatocellular Carcinoma. Gastroenterology, 2016. 150(4): 835-53.

18. Ghosh S, Banerjee S. Whole-genome mutational analysis: cause and effect of noncoding and structural mutations in liver cancer. HepatoBiliary Surg Nutr. 2017;6(1):57-59.

19. Charoentong P, Finotello F, Angelova M, et al. Pan-cancer Immunogenomic Analyses Reveal Genotype-Immunophenotype Relationships and Predictors of Response to Checkpoint Blockade. Cell Rep. 2017 Jan 3;18(1):248-262.

20. Long J, Chen P, Lin J, et al. DNA methylation-driven genes for constructing diagnostic, prognostic, and recurrence models for hepatocellular carcinoma. Theranostics. 2019;9:7251-67.

21. Marquardt JU, Galle PR, Teufel A. Molecular diagnosis and therapy of hepatocellular carcinoma (HCC): an emerging field for advanced technologies. J Hepatol. 2012;56:267-75.

22. Steuer CE, Ramalingam SS. Tumor mutation burden: leading immunotherapy to the era of precision medicine? J Clin Oncol. 2018;36:631-2.

\section{Figures}




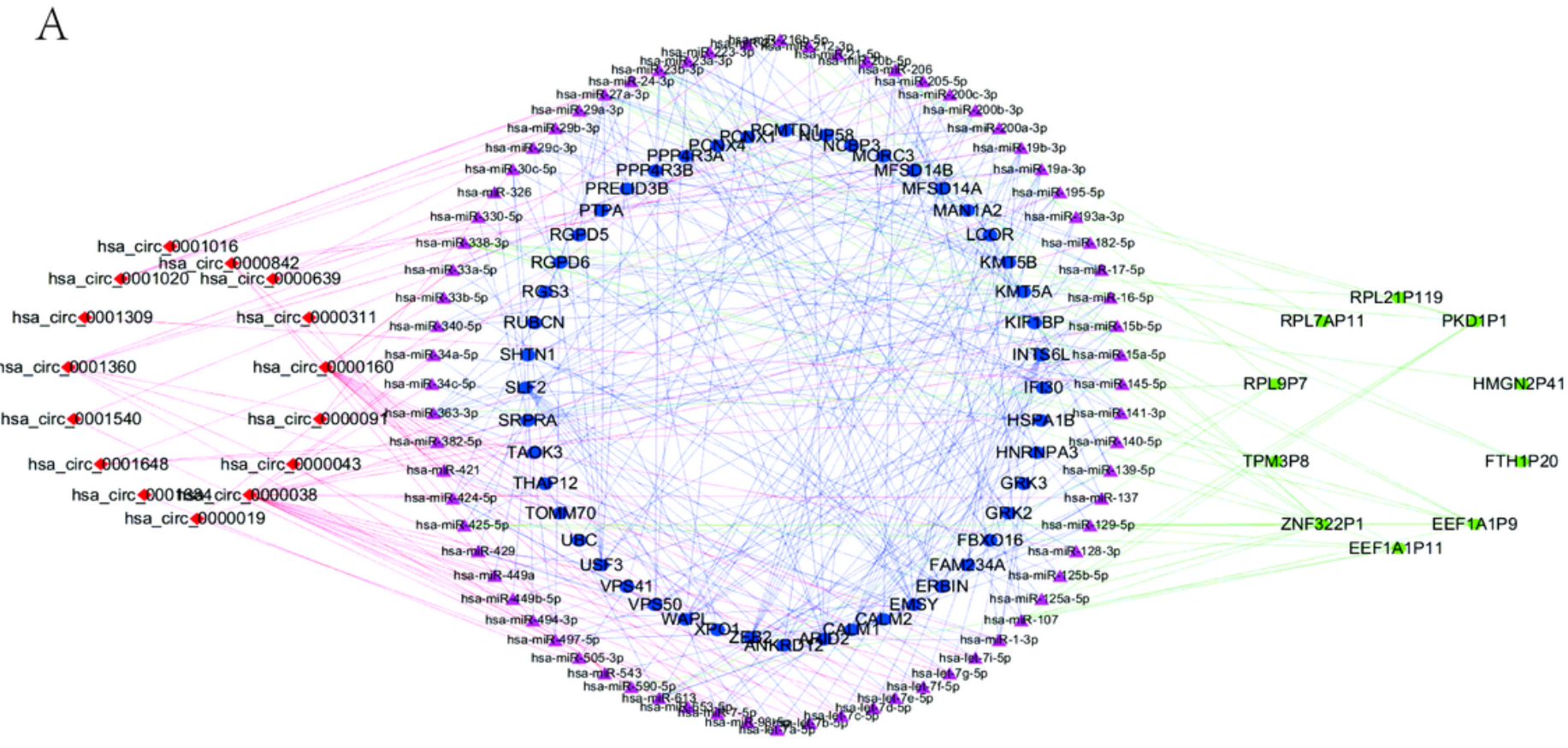

B

protein localization to nucleus positive regulation of phosphoprotein phosphatase activity
positive regulation of phosphatase activity nucleotide-binding oligomerization domain containing signaling pathwaynucleotide-binding domain, leucine rich repeat containing receptor signaling pathwaypositive regulation of protein dephosphorylation intracellular transport of virus positive regulation of dephosphorylation negative regulation of ryanodine-sensitive calcium-release channel activity

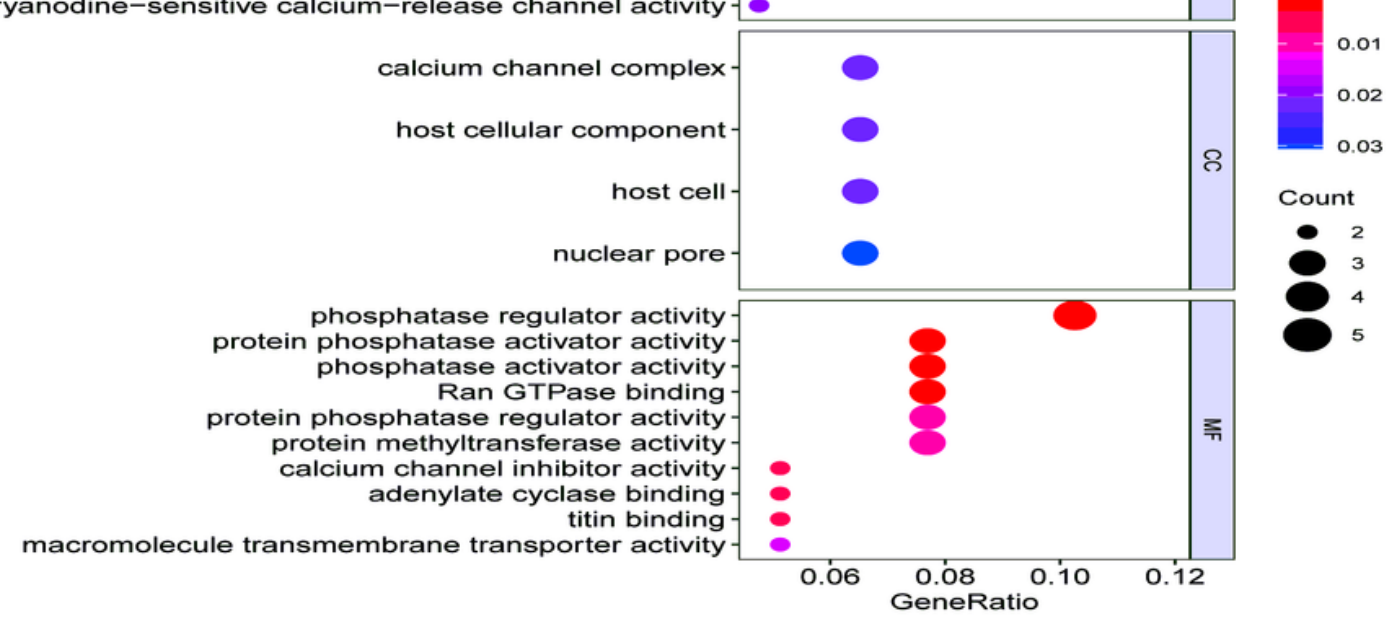

\section{Figure 1}

Construction of CeRNA network, GO enrichment analysis of exosome -related genes. (A) Construct CeRNA network from exoRbase database. (B) GO enrichment analysis of exosome-related genes. 
A
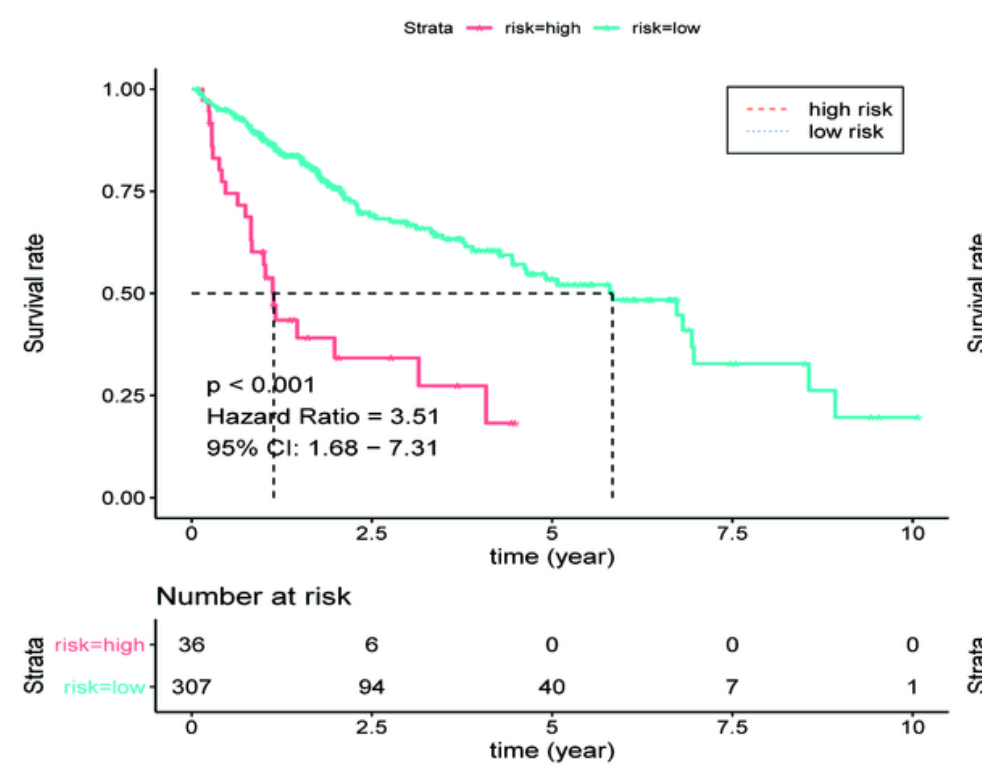

C

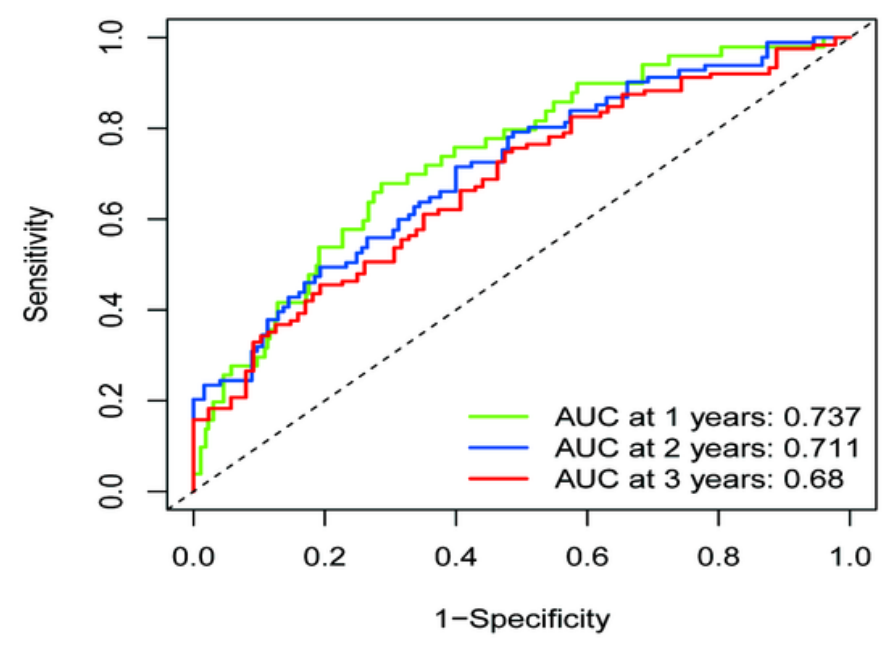

B
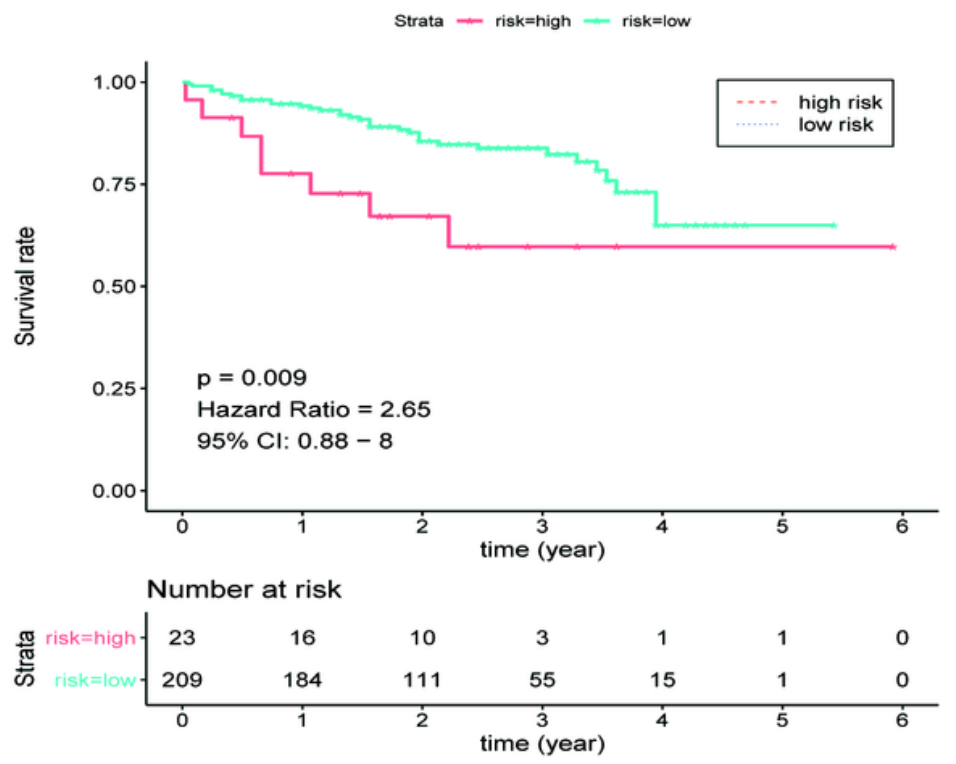

$\mathrm{D}$

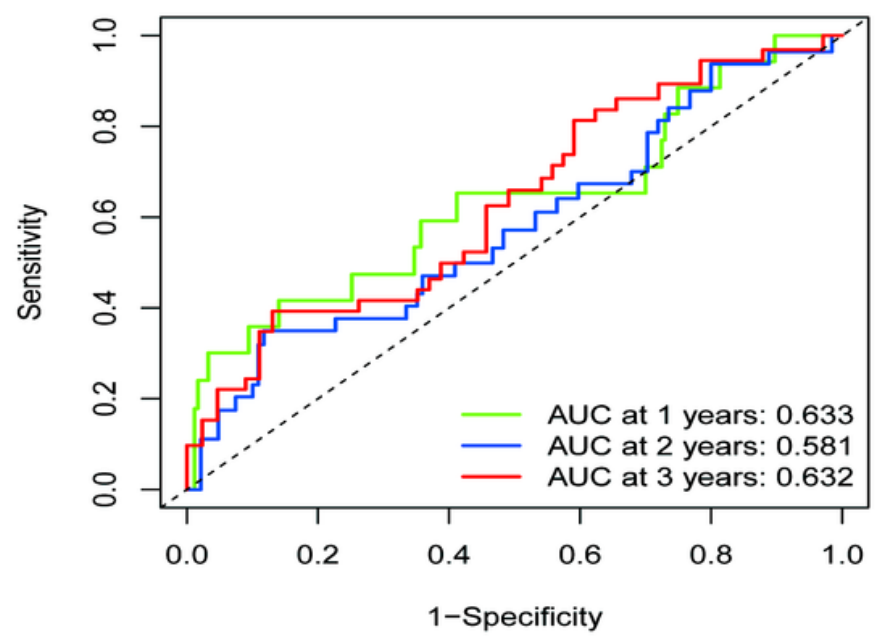

Figure 2

Establishment of a prognostic model of exosome-related genes in TCGA database. Evaluation of the performance of exosome-related prognostic model in ICGC dataset (A) The Kaplan-Meier survival curves of the five prognostic genes in the testing dataset. (B) The Kaplan-Meier survival curves of the five prognostic genes in the validation dataset. (C) 1-year, 2-year, and 3-year predicted time-dependent ROC of the five predicted genes in the testing dataset. (D) 1-year, 2-year, and 3-year predicted time-dependent ROC of the five predicted genes in the validation dataset. 
A

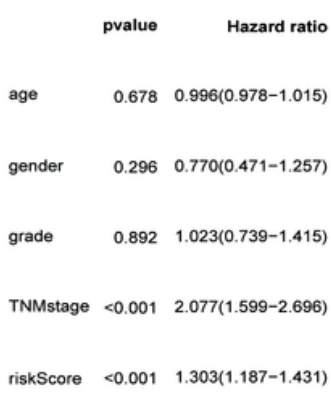

riskScore $\quad<0.001 \quad 1.303(1.187-1.431)$

$\mathrm{B}$
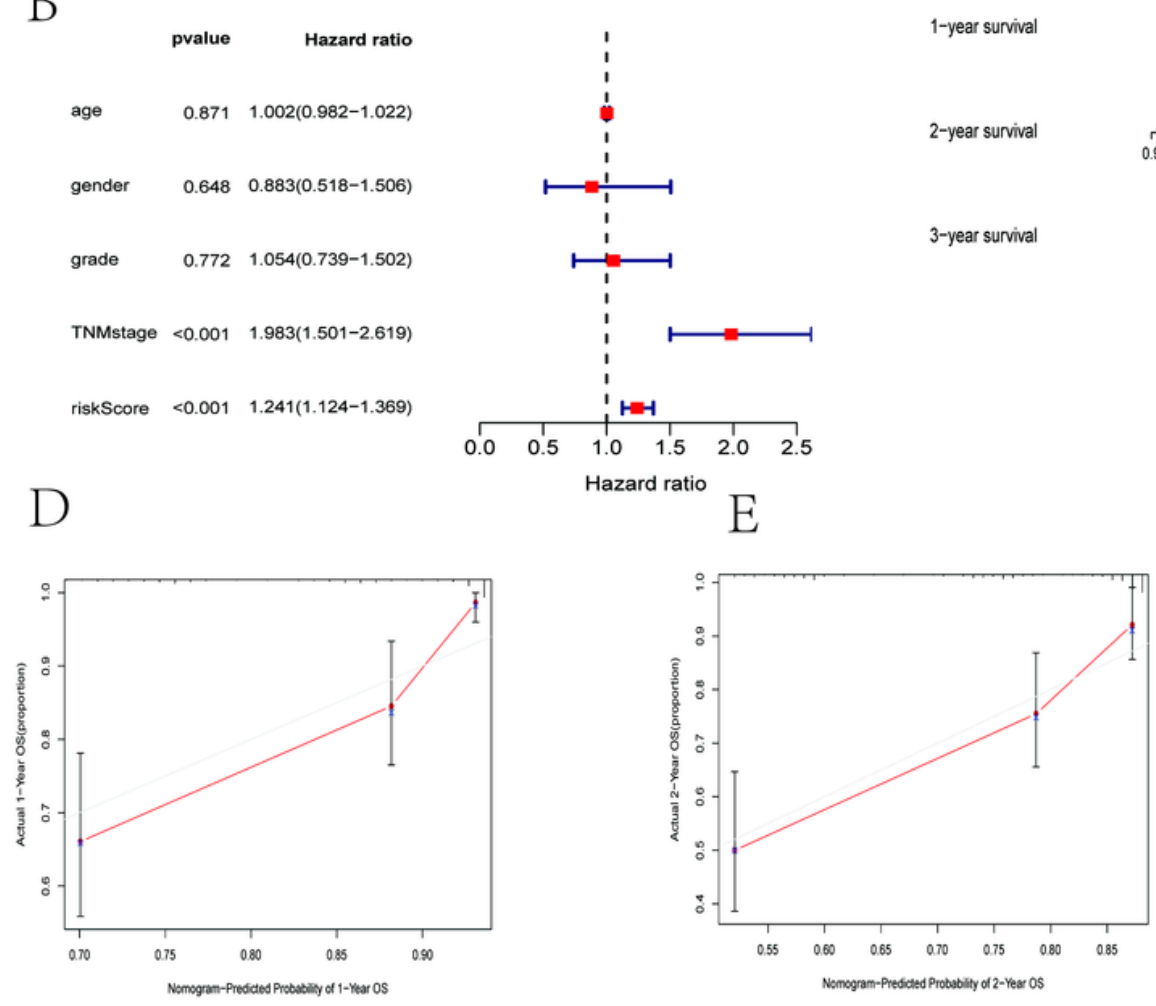

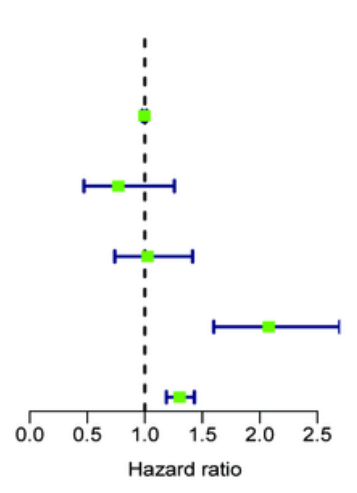

C

Points

TNMstage

riskScore

Total Points
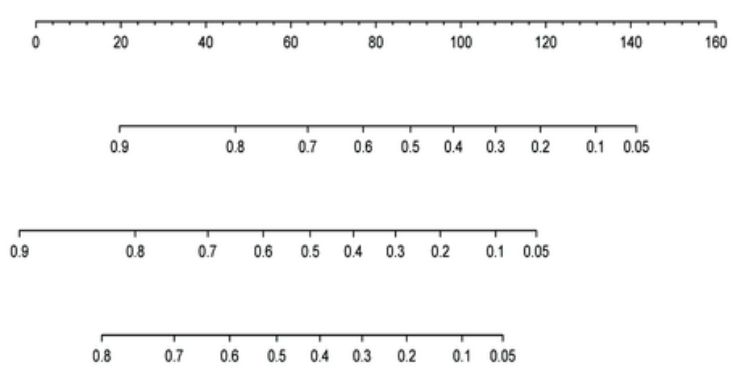

\section{Figure 3}

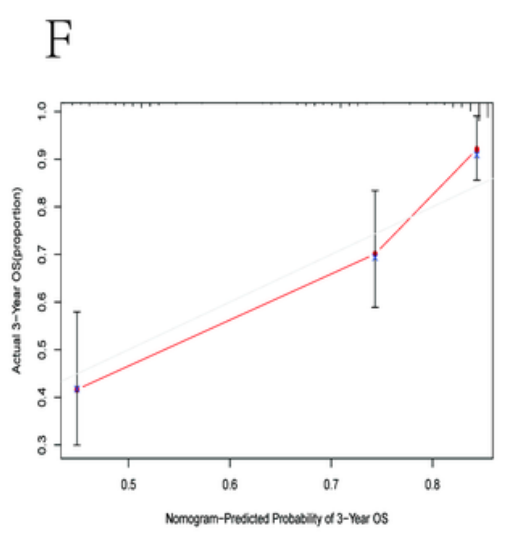

Construction of a predictive nomogram. (A) Forrest plot of the univariate Cox regression analysis in HCC. (B) Forrest plot of the multivariate Cox regression analysis in HCC. (C) Building the nomogram predicting overall survival for HCC patients in the TCGA cohort. The nomogram plot was built based on two independent prognostic factors in HCC.. 

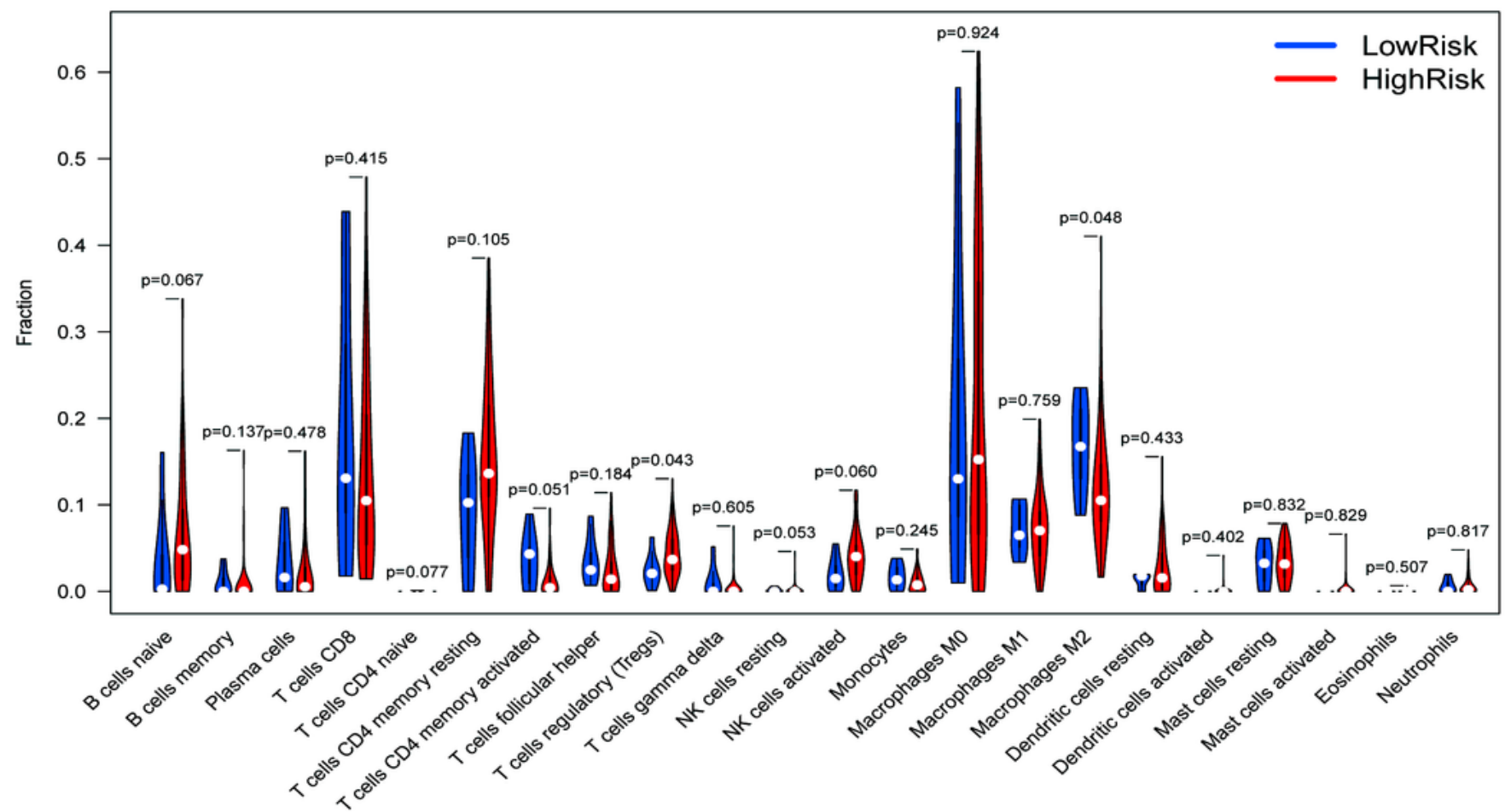

$\mathrm{B}$

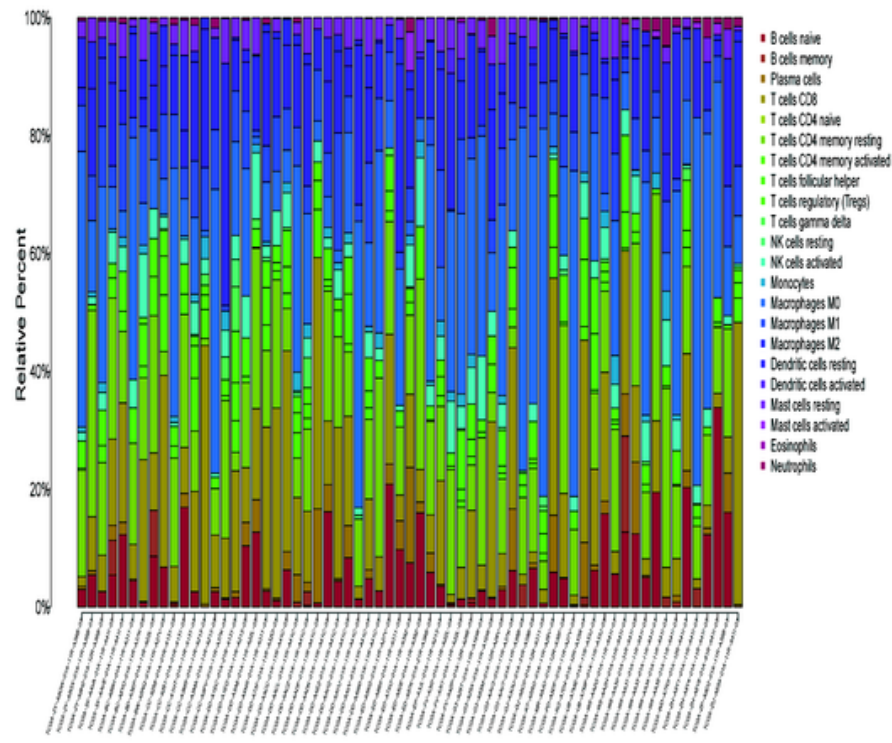

C

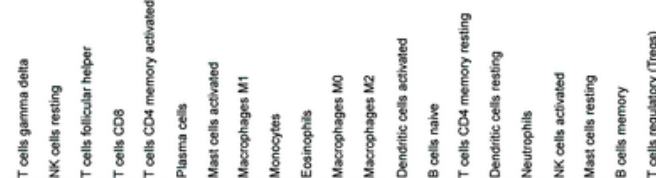

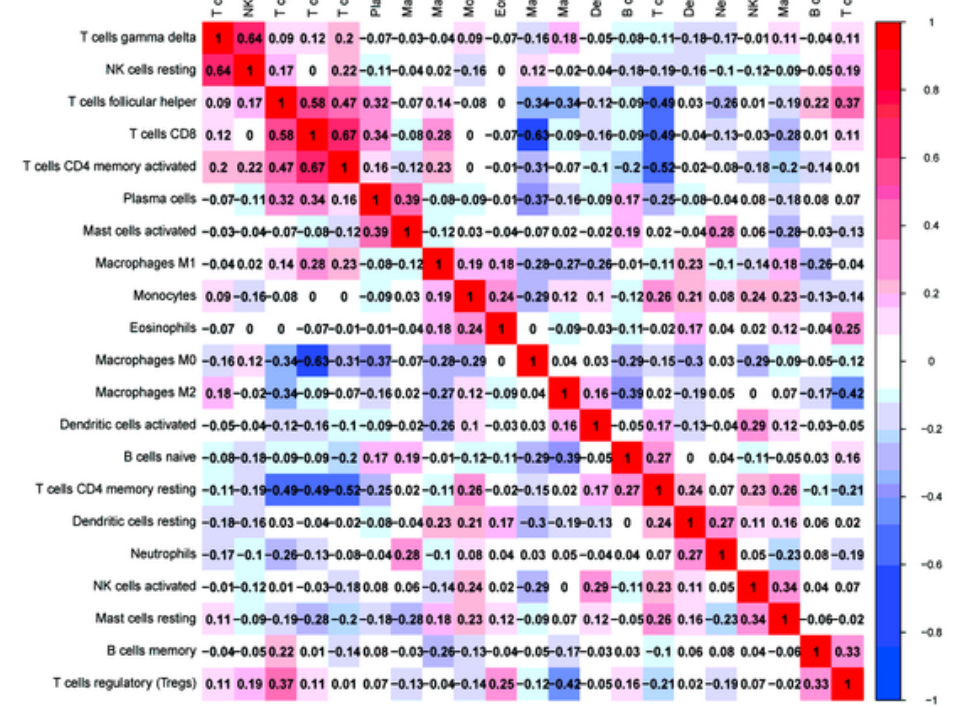

\section{Figure 4}

Relationship of the exosome-related prognostic signature and immune cell infiltration heterogeneity. (A) The difference of immune cell infiltration between low- and high-risk groups in HCC patients. (B) Heatmap displayed the relative infiltration of immune cell populations for tumor samples with available RNAsequencing data from TCGA dataset. (C) Heatmap showing the correlation between immune cells population. 

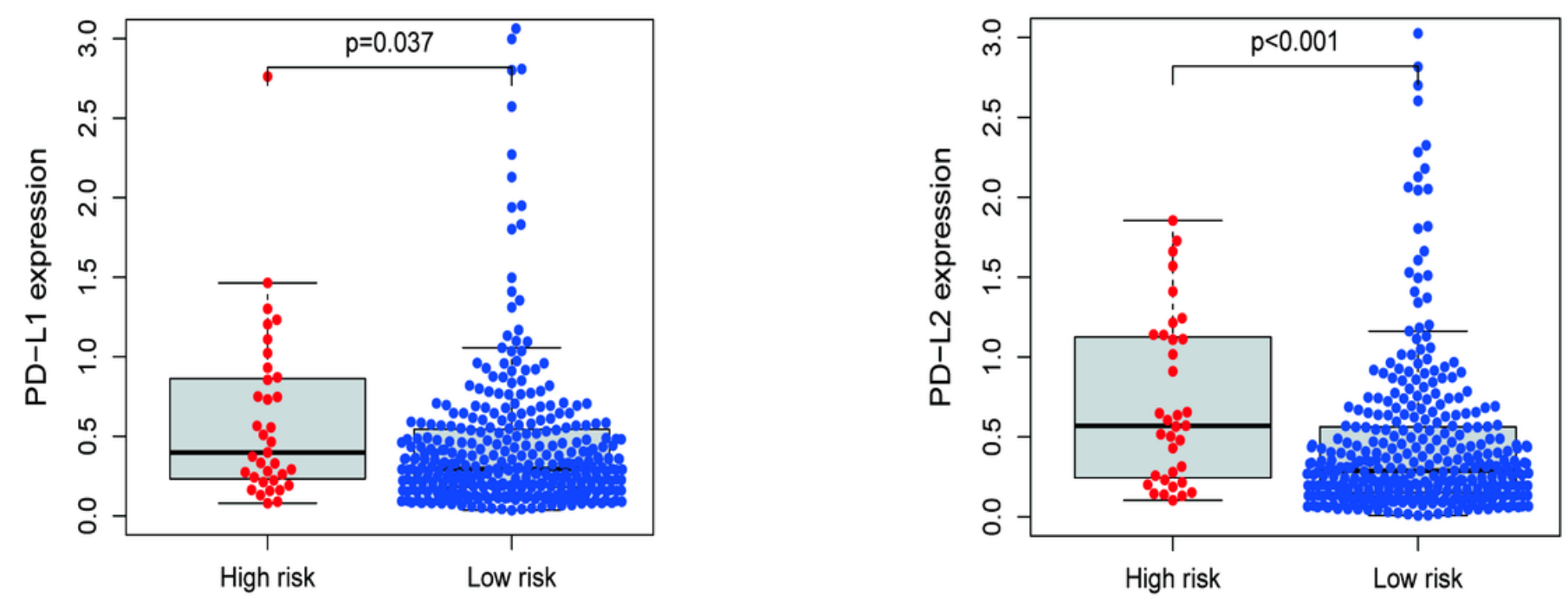

C

$D$
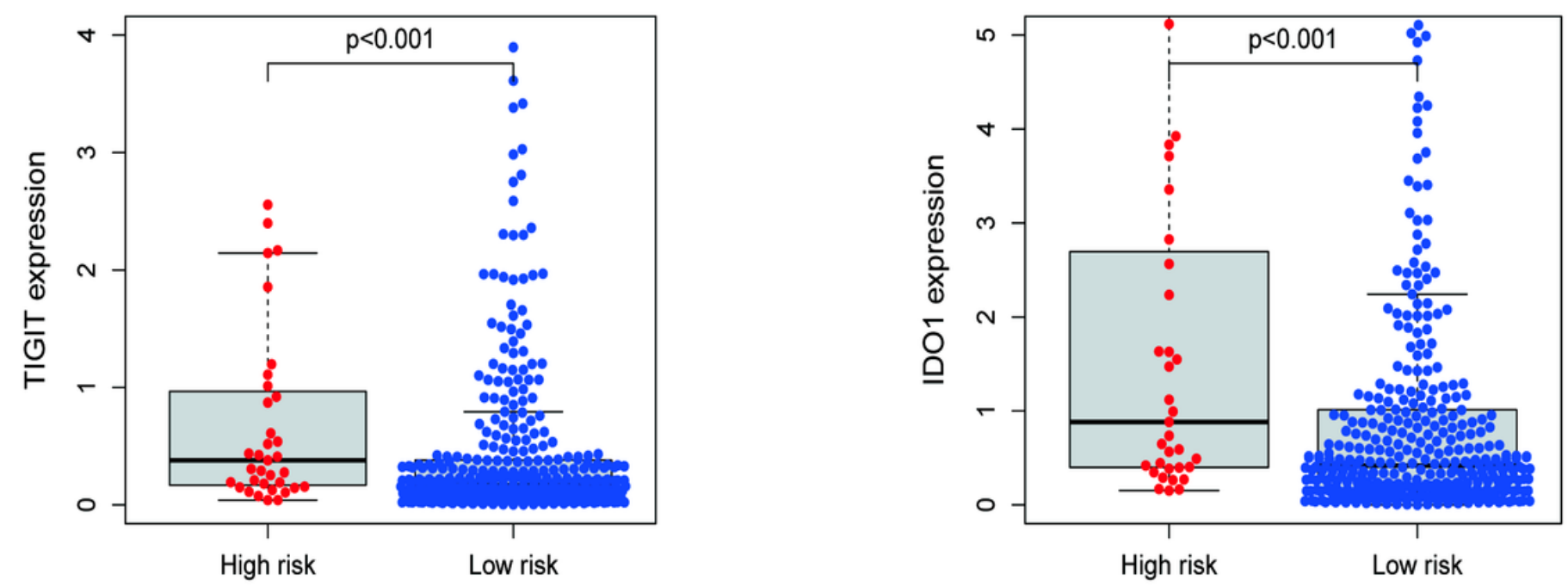

Figure 5

Differences in expression of immunomodulatory molecules between low- and high-risk groups.

Relationships between the low- and high-risk groups and difference in expression of PD-L1(A), PD-L2(B), TIGIT(C) and ID01(D).

\section{Supplementary Files}

This is a list of supplementary files associated with this preprint. Click to download. 
- SupplementalFigure1.tif

- SupplementalFigure2.tif

- SupplementalTable1.xlsx 